\title{
BLOWUP OF SOLUTIONS WITH POSITIVE ENERGY IN NONLINEAR THERMOELASTICITY WITH SECOND SOUND
}

\author{
SALIM A. MESSAOUDI AND BELKACEM SAID-HOUARI \\ Received 6 November 2003 and in revised form 14 April 2004
}

This work is concerned with a semilinear thermoelastic system, where the heat flux is given by Cattaneo's law instead of the usual Fourier's law. We will improve our earlier result by showing that the blowup can be obtained for solutions with "relatively" positive initial energy. Our technique of proof is based on a method used by Vitillaro with the necessary modifications imposed by the nature of our problem.

\section{Introduction}

Results concerning existence, blowup, and asymptotic behaviors of smooth, as well as weak, solutions in classical thermoelasticity have been established by several authors over the past two decades. See in this regard $[1,2,3,4,5,6,7,8,11,12,13,14,17,18,20]$.

For thermoelasticity with second sound, Tarabek [21] considered problems related to the one-dimensional system

$$
\begin{aligned}
u_{t t}-a\left(u_{x}, \theta, q\right) u_{x x}+b\left(u_{x}, \theta, q\right) \theta_{x} & =\alpha_{1}\left(u_{x}, \theta\right) q q_{x} \\
\theta_{t}+g\left(u_{x}, \theta, q\right) q_{x}+d\left(u_{x}, \theta, q\right) u_{t x} & =\alpha_{2}\left(u_{x}, \theta\right) q q_{t} \\
\tau\left(u_{x}, \theta\right) q_{t}+q+k\left(u_{x}, \theta\right) \theta_{x} & =0
\end{aligned}
$$

in both bounded and unbounded situations and established global existence results for small initial data. He also showed that these "classical" solutions tend to equilibrium as $t$ tends to infinity; however, no rate of decay has been discussed. In his work, Tarabek used the usual energy argument and exploited some relations from the second law of thermodynamics to overcome the difficulty arising from the lack of Poincare's inequality in the unbounded domains. Relations from thermodynamics have been also used by Hrusa \& Tarabek [4] to prove a global existence for the Cauchy problem to a classical thermoelasticity system and then by Hrusa \& Messaoudi [3] to establish a blowup result for a thermoelastic system. Saouli [19] used the nonlinear semigroup theory to prove a local existence result for a system similar to the one considered by Tarabek. 
Concerning the asymptotic behavior, Racke [15] discussed lately (1.1) and established exponential decay results for several linear and nonlinear initial boundary value problems. In particular, he studied the system (1.1) for a rigidly clamped medium with temperature held constant on the boundary, that is,

$$
u(t, 0)=u(t, 1)=\theta(t, 0)=\theta(t, 1)=\bar{\theta}, \quad t \geq 0,
$$

and showed that, for small enough initial data and for $\alpha_{1}=\alpha_{2}=0$, classical solutions decay exponentially to the equilibrium state. We should note here that, although the dissipative effects of heat conduction induced by Cattaneo's law are usually weaker than those induced by Fourier's law, a global existence as well as exponential decay results for small initial data have been established. For a discussion in this direction, see Racke [15]. Messaoudi and Said-Houari [10] extended lately the decay result of [15] to (1.1) for $\alpha_{1}$ and $\alpha_{2}$ that are not necessarily zero.

Regarding the multidimensional case $(n=2,3)$, Racke [16] established an existence result for the $n$-dimensional problem

$$
\begin{gathered}
u_{t t}-\mu \Delta u-(\mu+\lambda) \nabla \operatorname{div} u+\beta \nabla \theta=0, \\
\theta_{t}+\gamma \operatorname{div} q+\delta \operatorname{div} u_{t}=0, \\
\tau q_{t}+q+\kappa \nabla \theta=0, \quad x \in \Omega, \quad t>0, \\
u(\cdot, 0)=u_{0}, \quad u_{t}(\cdot, 0)=u_{1}, \quad \theta(\cdot, 0)=\theta_{0}, \quad q(\cdot, 0)=q_{0}, \quad x \in \Omega, \\
u=\theta=0, \quad x \in \partial \Omega, \quad t \geq 0,
\end{gathered}
$$

where $\Omega$ is a bounded domain of $\mathbb{R}^{n}$, with a smooth boundary $\partial \Omega, u=u(x, t) \in \mathbb{R}^{n}$ is the displacement vector, $\theta=\theta(x, t)$ is the difference temperature, $q=q(x, t) \in \mathbb{R}^{n}$ is the heat flux vector, and $\mu, \lambda, \beta, \gamma, \delta, \tau, \kappa$ are positive constants, where $\mu, \lambda$ are Lame moduli and $\tau$ is the relaxation time, a small parameter compared to the others. In particular, if $\tau=0$, (1.3) reduces to the system of classical thermoelasticity in which the heat flux is given by Fourier's law instead of Cattaneo's law. He also proved, under the conditions rot $u=$ $\operatorname{rot} q=0$, an exponential decay result for (1.3). This result includes the radially symmetric solution, as it is on only a special case. Messaoudi [9] investigated the situation where a nonlinear source term is competing with the damping caused by the heat conduction and established a local existence result. He also showed that solutions with negative energy blow up in finite time. His work generalized an earlier one in $[7,8]$ to thermoelasticity with second sound.

In this paper, we are concerned with the nonlinear problem

$$
\begin{gathered}
u_{t t}-\mu \Delta u-(\mu+\lambda) \nabla \operatorname{div} u+\beta \nabla \theta=|u|^{p-2} u, \\
\theta_{t}+\gamma \operatorname{div} q+\delta \operatorname{div} u_{t}=0, \\
\tau q_{t}+q+\kappa \nabla \theta=0, \quad x \in \Omega, \quad t>0, \\
u(\cdot, 0)=u_{0}, \quad u_{t}(\cdot, 0)=u_{1}, \quad \theta(\cdot, 0)=\theta_{0}, \quad q(\cdot, 0)=q_{0}, \quad x \in \Omega, \\
u=\theta=0, \quad x \in \partial \Omega, \quad t \geq 0,
\end{gathered}
$$

for $p>2$. This is a similar problem to (1.3), with a nonlinear source term competing with the damping factor. We will extend the blowup result of [9] to situations where the energy 
can be positive. Our technique of proof follows carefully the techniques of Vitillaro [22] with the necessary modifications imposed by the nature of our problem. For the sake of completeness, we state here the local existence of [9]. For this purpose, we introduce the following functional spaces:

$$
\begin{aligned}
& \Pi:=\left[H_{0}^{1}(\Omega) \cap H^{2}(\Omega)\right]^{n} \times\left[H_{0}^{1}(\Omega)\right]^{n} \times H_{0}^{1}(\Omega) \times D, \\
& D:=\left\{q \in\left[L^{2}(\Omega)\right]^{n} \text { such that } \operatorname{div} q \in L^{2}(\Omega)\right\} \\
& H:=\left[H_{0}^{1}(\Omega)\right]^{n} \times\left[L^{2}(\Omega)\right]^{n} \times L^{2}(\Omega) \times\left[L^{2}(\Omega)\right]^{n} .
\end{aligned}
$$

Theorem 1.1. Assume that

$$
\begin{gathered}
2<p \leq \frac{2(n-3)}{n-4}, \quad n \geq 5, \\
2<p, \quad n \leq 4,
\end{gathered}
$$

holds. Then given any $\left(u_{0}, u_{1}, \theta_{0}, q_{0}\right) \in \Pi$, there exists a positive number $T$ small enough such that problem (1.4) has a unique strong solution satisfying

$$
\left(u, u_{t}, \theta, q\right) \in C^{1}([0, T) ; \Pi) \cap C([0, T) ; H) .
$$

\section{Blowup}

In order to state and prove our result we introduce the following: let $B_{1}$ be the best constant of the Sobolev imbedding $\left[H_{0}^{1}(\Omega)\right]^{n} \hookrightarrow\left[L^{p}(\Omega)\right]^{n}$ and $B_{2}=B_{1} / \mu$. We set

$$
\begin{gathered}
\alpha_{1}=B_{2}^{-p /(p-2)}, \quad E_{1}=\left(\frac{1}{2}-\frac{1}{p}\right) \alpha_{1}^{2}, \\
E(t)=\frac{1}{2}\left\|u_{t}\right\|_{2}^{2}+\frac{\mu}{2}\|\nabla u\|_{2}^{2}+\frac{\lambda+\mu}{2}\|\operatorname{div} u\|_{2}^{2}+\frac{\beta}{2 \delta}\|\theta\|_{2}^{2}+\frac{\gamma \beta \tau}{2 \delta k}\|q\|_{2}^{2}-\frac{1}{p}\|u\|_{p}^{p}
\end{gathered}
$$

Lemma 2.1. Let $(u, \theta, q)$ be solution of (1.4). Assume that $E(0)<E_{1}$ and

$$
\left[\mu\left\|\nabla u_{0}\right\|_{2}^{2}+(\lambda+\mu)\left\|\operatorname{div} u_{0}\right\|_{2}^{2}+\frac{\beta}{\delta}\left\|\theta_{0}\right\|_{2}^{2} \frac{\gamma \beta \tau}{\delta k}\left\|q_{0}\right\|_{2}^{2}\right]^{1 / 2}>B_{2}^{-p /(p-2)}
$$

Then there exists a constant $\alpha_{2}>B_{2}^{-p /(p-2)}$ such that

$$
\begin{gathered}
{\left[\mu\|\nabla u\|_{2}^{2}+(\lambda+\mu)\|\operatorname{div} u\|_{2}^{2}+\frac{\beta}{\delta}\|\theta\|_{2}^{2}+\frac{\gamma \beta \tau}{\delta k}\|q\|_{2}^{2}\right]^{1 / 2} \geq \alpha_{2},} \\
\|u\|_{p} \geq B_{2} \alpha_{2}, \quad \forall t \in[0, T) .
\end{gathered}
$$


204 Blowup in thermoelasticity with second sound

Proof. We first note that, by (2.2) and the Sobolev imbedding, we have

$$
\begin{aligned}
E(t) \geq & \frac{\mu}{2}\|\nabla u\|_{2}^{2}+\frac{\lambda+\mu}{2}\|\operatorname{div} u\|_{2}^{2}+\frac{\beta}{2 \delta}\|\theta\|_{2}^{2}+\frac{\gamma \beta \tau}{2 \delta k}\|q\|_{2}^{2}-\frac{1}{p}\|u\|_{p}^{p} \\
\geq & \frac{1}{2}\left[\mu\|\nabla u\|_{2}^{2}+(\lambda+\mu)\|\operatorname{div} u\|_{2}^{2}+\frac{\beta}{\delta}\|\theta\|_{2}^{2}+\frac{\gamma \beta \tau}{\delta k}\|q\|_{2}^{2}\right] \\
& -\frac{B_{2}^{p}}{p}\left[\mu\|\nabla u\|_{2}^{2}+(\lambda+\mu)\|\operatorname{div} u\|_{2}^{2}+\frac{\beta}{\delta}\|\theta\|_{2}^{2}+\frac{\gamma \beta \tau}{\delta k}\|q\|_{2}^{2}\right]^{p / 2} \\
= & \frac{1}{2} \alpha^{2}-\frac{B_{2}^{p}}{p} \alpha^{p}=g(\alpha),
\end{aligned}
$$

where

$$
\alpha=\left[\mu\|\nabla u\|_{2}^{2}+(\lambda+\mu)\|\operatorname{div} u\|_{2}^{2}+\frac{\beta}{\delta}\|\theta\|_{2}^{2}+\frac{\gamma \beta \tau}{\delta k}\|q\|_{2}^{2}\right]^{1 / 2} .
$$

It is easy to verify that $g$ is increasing for $0<\alpha<\alpha_{1}$, decreasing for $\alpha>\alpha_{1}, g(\alpha) \rightarrow-\infty$ as $\alpha \rightarrow+\infty$, and

$$
g\left(\alpha_{1}\right)=\left(\frac{1}{2}-\frac{1}{p}\right) B_{2}^{-2 p /(p-2)}=E_{1}
$$

where $\alpha_{1}$ is given in (2.1). Therefore, since $E(0)<E_{1}$, there exists $\alpha_{2}>\alpha_{1}$ such that $g\left(\alpha_{2}\right)=$ $E(0)$.

If we set

$$
\alpha_{0}=\left[\mu\left\|\nabla u_{0}\right\|_{2}^{2}+(\lambda+\mu)\left\|\operatorname{div} u_{0}\right\|_{2}^{2}+\frac{\beta}{\delta}\left\|\theta_{0}\right\|_{2}^{2}+\frac{\gamma \beta \tau}{\delta k}\left\|q_{0}\right\|_{2}^{2}\right]^{1 / 2},
$$

then by (2.6), we have

$$
g\left(\alpha_{0}\right) \leq E(0)=g\left(\alpha_{2}\right)
$$

which implies that $\alpha_{0} \geq \alpha_{2}$.

Now to establish (2.4), we suppose by contradiction that

$$
\left[\mu\left\|\nabla u\left(t_{0}\right)\right\|_{2}^{2}+(\lambda+\mu)\left\|\operatorname{div} u\left(t_{0}\right)\right\|_{2}^{2}+\frac{\beta}{\delta}\left\|\theta\left(t_{0}\right)\right\|_{2}^{2}+\frac{\gamma \beta \tau}{\delta k}\left\|q\left(t_{0}\right)\right\|_{2}^{2}\right]^{1 / 2}<\alpha_{2}
$$

for some $t_{0}>0$ and by the continuity of

$$
\mu\|\nabla u(\cdot)\|_{2}^{2}+(\lambda+\mu)\|\operatorname{div} u(\cdot)\|_{2}^{2}+\frac{\beta}{\delta}\|\theta(\cdot)\|_{2}^{2} \frac{\gamma \beta \tau}{\delta k}\|q(\cdot)\|_{2}^{2}
$$

we can choose $t_{0}$ such that

$$
\left[\mu\left\|\nabla u\left(t_{0}\right)\right\|_{2}^{2}+(\lambda+\mu)\left\|\operatorname{div} u\left(t_{0}\right)\right\|_{2}^{2}+\frac{\beta}{\delta}\left\|\theta\left(t_{0}\right)\right\|_{2}^{2} \frac{\gamma \beta \tau}{\delta k}\left\|q\left(t_{0}\right)\right\|_{2}^{2}\right]^{1 / 2}>\alpha_{1} .
$$


Again the use of (2.6) leads to

$$
E\left(t_{0}\right) \geq g\left(\mu\left\|\nabla u\left(t_{0}\right)\right\|_{2}^{2}+(\lambda+\mu)\left\|\operatorname{div} u\left(t_{0}\right)\right\|_{2}^{2}+\frac{\beta}{\delta}\left\|\theta\left(t_{0}\right)\right\|_{2}^{2} \frac{\gamma \beta \tau}{\delta k}\left\|q\left(t_{0}\right)\right\|_{2}^{2}\right)>g\left(\alpha_{2}\right)=E(0) .
$$

This is impossible since $E(t) \leq E(0)$ for all $t \in[0, T)$. Hence (2.4) is established.

To prove (2.5), we exploit (2.2) to see

$$
\frac{1}{2}\left[\mu\|\nabla u\|_{2}^{2}+(\lambda+\mu)\|\operatorname{div} u\|_{2}^{2}+\frac{\beta}{\delta}\|\theta\|_{2}^{2} \frac{\gamma \beta \tau}{\delta k}\|q\|_{2}^{2}\right] \leq E(0)+\frac{1}{p}\|u\|_{p}^{p} .
$$

Consequently,

$$
\begin{aligned}
\frac{1}{p}\|u\|_{p}^{p} & \geq \frac{1}{2}\left[\mu\|\nabla u\|_{2}^{2}+(\lambda+\mu)\|\operatorname{div} u\|_{2}^{2}+\frac{\beta}{\delta}\|\theta\|_{2}^{2}+\frac{\gamma \beta \tau}{\delta k}\|q\|_{2}^{2}\right]-E(0) \\
& \geq \frac{1}{2} \alpha_{2}^{2}-E(0) \geq \frac{1}{2} \alpha_{2}^{2}-g\left(\alpha_{2}\right)=\frac{B_{2}^{p}}{p} \alpha_{2}^{p}
\end{aligned}
$$

Therefore, (2.16) and (2.1) yield the desired result.

Theorem 2.2. Suppose that

$$
\begin{gathered}
2<p \leq \frac{2 n}{n-2}, \quad n \geq 3, \\
\frac{\beta \tau \delta}{\kappa \gamma}<8 .
\end{gathered}
$$

Then any solution of (1.4), with initial data satisfying

$$
\left[\mu\left\|\nabla u_{0}\right\|_{2}^{2}+(\lambda+\mu)\left\|\operatorname{div} u_{0}\right\|_{2}^{2}+\frac{\beta}{\delta}\left\|\theta_{0}\right\|_{2}^{2}+\frac{\gamma \beta \tau}{\delta k}\left\|q_{0}\right\|_{2}^{2}\right]>B_{2}^{-2 p /(p-2)}
$$

and

$$
E(0)<E_{1}
$$

blows up in finite time.

Remark 2.3. The condition (2.18) is "physically" reasonable due to the very small value of $\tau$. For instance, in [15], for the isotropic silicon and a medium temperature of $300 \mathrm{~K}$, we have

$$
\begin{gathered}
\beta \approx 391.62\left[\frac{m^{2}}{s^{2} K}\right], \quad \tau \approx 10^{-12}[s], \quad \delta \approx 163.82[K], \\
\gamma \approx 5.99 \times 10^{-7}\left[\frac{m s^{2} K}{k g}\right], \quad \kappa \approx 148\left[\frac{W}{m K}\right]
\end{gathered}
$$

consequently, we get

$$
\frac{\beta \tau \delta}{\kappa \gamma} \approx 72.367 \times 10^{-7}<8
$$


206 Blowup in thermoelasticity with second sound

Proof. A multiplication of (1.4) by $u_{t},(\beta / \delta) \theta$, and $(\beta \gamma / \delta \tau) q$, respectively, integration over $\Omega$, using integration by parts, and addition of equalities yields

$$
E^{\prime}(t)=-\frac{\gamma \beta}{\delta k}\|q\|_{2}^{2} \leq 0
$$

We then set

$$
H(t)=E_{1}-E(t)
$$

By using (2.2) and (2.23), we get

$$
\begin{aligned}
0 & <H(0) \leq H(t) \\
& \leq E_{1}-\frac{1}{2}\left(\left\|u_{t}\right\|_{2}^{2}+\mu\|\nabla u\|_{2}^{2}+(\lambda+\mu)\|\operatorname{div} u\|_{2}^{2}+\frac{\beta}{\delta}\|\theta\|_{2}^{2}+\frac{\gamma \beta \tau}{\delta k}\|q\|_{2}^{2}\right)+\frac{1}{p}\|u\|_{p}^{p},
\end{aligned}
$$

and from (2.1) and (2.4), we obtain

$$
\begin{aligned}
& E_{1}- \frac{1}{2}\left(\left\|u_{t}\right\|_{2}^{2}+\mu\|\nabla u\|_{2}^{2}+(\lambda+\mu)\|\operatorname{div} u\|_{2}^{2}+\frac{\beta}{\delta}\|\theta\|_{2}^{2}+\frac{\gamma \beta \tau}{\delta k}\|q\|_{2}^{2}\right) \\
&<E_{1}-\frac{1}{2} \alpha_{1}^{2}=-\frac{1}{p} \alpha_{1}^{2}<0, \quad \forall t \geq 0 .
\end{aligned}
$$

Hence

$$
0<H(0) \leq H(t) \leq \frac{1}{p}\|u\|_{p}^{p}, \quad \forall t \geq 0
$$

We then define

$$
L(t)=H^{1-\sigma}(t)+\varepsilon \int_{\Omega}\left[u \cdot u_{t}+\frac{\beta \tau}{k} u \cdot q\right](x, t) d x
$$

for $\varepsilon$ small to be chosen later and

$$
\sigma=\frac{p-2}{2 p} .
$$

By taking a derivative of (2.28) and using (1.4), we obtain

$$
\begin{aligned}
L^{\prime}(t)= & (1-\sigma) H^{-\sigma}(t) H^{\prime}(t)+\varepsilon\left(\|u\|_{p}^{p}+\left\|u_{t}\right\|_{2}^{2}-\mu\|\nabla u\|_{2}^{2}-(\lambda+\mu)\|\operatorname{div} u\|_{2}^{2}\right) \\
& -\frac{\varepsilon \beta}{k} \int_{\Omega} u \cdot q d x+\frac{\varepsilon \beta \tau}{k} \int_{\Omega} u_{t} \cdot q d x .
\end{aligned}
$$

By exploiting (2.2) and (2.24), the estimate (2.30) takes the form

$$
\begin{aligned}
L^{\prime}(t)= & (1-\sigma) H^{-\sigma}(t) H^{\prime}(t)+\varepsilon\left(1-\frac{2}{p}\right)\|u\|_{p}^{p}+2 \varepsilon\left\|u_{t}\right\|_{2}^{2}-\frac{\varepsilon \beta}{k} \int_{\Omega} u \cdot q d x \\
& +\frac{\varepsilon \beta \tau}{k} \int_{\Omega} u_{t} \cdot q d x+2 \varepsilon H(t)-2 \varepsilon E_{1}+\frac{\varepsilon \beta}{\delta}\|\theta\|_{2}^{2}+\frac{\varepsilon \gamma \beta \tau}{\delta k}\|q\|_{2}^{2} .
\end{aligned}
$$


Then using (2.5), we obtain

$$
\begin{aligned}
L^{\prime}(t) \geq & (1-\sigma) H^{-\sigma}(t) H^{\prime}(t)+\varepsilon\left(1-\frac{2}{p}-2 E_{1}\left(B_{2} \alpha_{2}\right)^{-p}\right)\|u\|_{p}^{p}+2 \varepsilon\left\|u_{t}\right\|_{2}^{2} \\
& -\frac{\varepsilon \beta}{k} \int_{\Omega} u \cdot q d x+\frac{\varepsilon \beta \tau}{k} \int_{\Omega} u_{t} \cdot q d x+2 \varepsilon H(t)+\frac{\varepsilon \beta}{\delta}\|\theta\|_{2}^{2}+\frac{\varepsilon \gamma \beta \tau}{\delta k}\|q\|_{2}^{2}
\end{aligned}
$$

which implies

$$
\begin{aligned}
L^{\prime}(t) \geq & (1-\sigma) H^{-\sigma}(t) H^{\prime}(t)+\varepsilon c_{0}\|u\|_{p}^{p}+2 \varepsilon\left\|u_{t}\right\|_{2}^{2}+2 \varepsilon H(t)+\frac{\varepsilon \beta}{\delta}\|\theta\|_{2}^{2} \\
& +\frac{\varepsilon \gamma \beta \tau}{\delta k}\|q\|_{2}^{2}-\frac{\varepsilon \beta}{k} \int_{\Omega} u \cdot q d x+\frac{\varepsilon \beta \tau}{k} \int_{\Omega} u_{t} \cdot q d x
\end{aligned}
$$

where $c_{0}=1-2 / p-2 E_{1}\left(B_{2} \alpha_{2}\right)^{-p}>0$ since $\alpha_{2}>B_{2}^{-p /(p-2)}$.

Next we exploit Young's inequality to estimate the last two terms in (2.33) as follows:

$$
\begin{aligned}
& \left|\int_{\Omega} u_{t} \cdot q d x\right| \leq \frac{a}{2}\left\|u_{t}\right\|_{2}^{2}+\frac{1}{2 a}\|q\|_{2}^{2}, \quad \forall a>0, \\
& \int_{\Omega} u \cdot q d x \leq \frac{b}{2}\|q\|_{2}^{2}+\frac{1}{2 b}\|u\|_{2}^{2}, \quad \forall b>0 .
\end{aligned}
$$

Thus (2.33) yields

$$
\begin{aligned}
L^{\prime}(t) \geq & (1-\sigma) H^{-\sigma}(t) H^{\prime}(t)+\varepsilon c_{0}\|u\|_{p}^{p}+\varepsilon\left(2-\frac{a \beta \tau}{2 k}\right)\left\|u_{t}\right\|_{2}^{2} \\
& +2 \varepsilon H(t)+\frac{\varepsilon \beta}{\delta}\|\theta\|_{2}^{2}+\varepsilon\left(\frac{\gamma \beta \tau}{\delta k}-\frac{\beta \tau}{2 a k}\right)\|q\|_{2}^{2}-\frac{\varepsilon \beta}{k}\left[\frac{b}{2}\|q\|_{2}^{2}+\frac{1}{2 b}\|u\|_{2}^{2}\right] .
\end{aligned}
$$

At this point, we choose $a$ so that

$$
A_{1}:=2-\frac{a \beta \tau}{2 k}>0, \quad A_{2}:=\frac{\beta \tau}{2 k}\left(\frac{2 \gamma}{\delta}-\frac{1}{a}\right)>0
$$

This is possible by virtue of (2.18); consequently, (2.35) becomes

$$
\begin{aligned}
L^{\prime}(t) \geq & (1-\sigma) H^{-\sigma}(t) H^{\prime}(t)+\varepsilon A_{1}\left\|u_{t}\right\|_{2}^{2}+\varepsilon A_{2}\|q\|_{2}^{2} \\
& +\varepsilon \mathcal{c}_{0}\|u\|_{p}^{p}+\varepsilon A_{3}\|\theta\|_{2}^{2}+2 \varepsilon H(t)-\frac{\varepsilon \beta}{k}\left[\frac{b}{2}\|q\|_{2}^{2}+\frac{1}{2 b} \| u_{2}^{2}\right],
\end{aligned}
$$

where $A_{1}, A_{2}, A_{3}$ are strictly positive constants. 
208 Blowup in thermoelasticity with second sound

We also set $b=2 M \gamma H^{-\sigma}(t) / \delta$, for $M$ a large constant to be determined, to deduce from (2.37)

$$
\begin{aligned}
L^{\prime}(t) \geq & {[(1-\sigma)-\varepsilon M] H^{-\sigma}(t) H^{\prime}(t)+\varepsilon A_{1}\left\|u_{t}\right\|_{2}^{2}+\varepsilon A_{2}\|q\|_{2}^{2} } \\
& +\varepsilon c_{0}\|u\|_{p}^{p}+\varepsilon A_{3}\|\theta\|_{2}^{2}+2 \varepsilon H(t)-\frac{C \varepsilon}{4 M} H^{\sigma}(t)\|u\|_{p}^{2},
\end{aligned}
$$

where $C$, here and in the sequel, is a positive generic constant depending on $\Omega, p, \beta, \gamma, \delta$, $k, \lambda, \mu, \tau$ only.

We then use (2.27) to get

$$
\begin{aligned}
L^{\prime}(t) \geq & {[(1-\sigma)-\varepsilon M] H^{-\sigma}(t) H^{\prime}(t)+\varepsilon A_{1}\left\|u_{t}\right\|_{2}^{2}+\varepsilon A_{2}\|q\|_{2}^{2} } \\
& +\varepsilon c_{0}\|u\|_{p}^{p}+\varepsilon A_{3}\|\theta\|_{2}^{2}+2 \varepsilon H(t)-\frac{C \varepsilon}{4 p M}\|u\|_{p}^{2+\sigma p} .
\end{aligned}
$$

By using (2.29) and the inequality

$$
z^{\nu} \leq(z+1) \leq\left(1+\frac{1}{a}\right)(z+a), \quad \forall z \geq 0,0<v \leq 1, a>0
$$

we have the following:

$$
\|u\|_{p}^{2+\sigma p} \leq d\left(\|u\|_{p}^{p}+H(0)\right) \leq d\left(\|u\|_{p}^{p}+H(t)\right), \quad \forall t \geq 0
$$

where $d=1+1 / H(0)$.

Inserting the estimate (2.41) into (2.39), we arrive at

$$
\begin{aligned}
L^{\prime}(t) \geq & {[(1-\sigma)-\varepsilon M] H^{-\sigma}(t) H^{\prime}(t)+\varepsilon A_{1}\left\|u_{t}\right\|_{2}^{2} } \\
& +\varepsilon A_{2}\|q\|_{2}^{2}+\varepsilon\left(c_{0}-\frac{C d}{4 p M}\right)\|u\|_{p}^{p}+\varepsilon A_{3}\|\theta\|_{2}^{2}+\varepsilon\left(2-\frac{C d}{4 p M}\right) H(t) .
\end{aligned}
$$

At this point, we choose $M$ large enough so that (2.42) becomes, for some positive constant $A_{0}$,

$$
L^{\prime}(t) \geq[(1-\sigma)-\varepsilon M] H^{-\sigma}(t) H^{\prime}(t)+\varepsilon A_{0}\left[\left\|u_{t}\right\|_{2}^{2}+\|q\|_{2}^{2}+\|u\|_{p}^{p}+H(t)\right]
$$

Once $M$ is fixed (hence $A_{0}$ ), we pick $\varepsilon$ small enough so that $(1-\sigma)-\varepsilon M \geq 0$ and

$$
L(0)=H^{1-\sigma}(0)+\varepsilon \int_{\Omega}\left[u_{0} \cdot u_{1}+\frac{\beta \tau}{k} u_{0} \cdot q_{0}\right](x, t) d x>0 .
$$


Therefore, (2.43) yields

$$
L^{\prime}(t) \geq \varepsilon A_{0}\left[\left\|u_{t}\right\|_{2}^{2}+\|q\|_{2}^{2}+\|u\|_{p}^{p}+H(t)\right]
$$

Consequently, we have

$$
L(t) \geq L(0)>0, \quad \forall t \geq 0 .
$$

Next we estimate

$$
\left|\int_{\Omega} u u_{t}(x, t) d x\right| \leq\|u\|_{2}\left\|u_{t}\right\|_{2} \leq C\|u\|_{p}\left\|u_{t}\right\|_{2}
$$

which implies

$$
\left|\int_{\Omega} u u_{t}(x, t) d x\right|^{1 /(1-\sigma)} \leq C\|u\|_{p}^{1 /(1-\sigma)}\left\|u_{t}\right\|_{2}^{1 /(1-\sigma)}
$$

Again Young's inequality gives us

$$
\left|\int_{\Omega} u u_{t}(x, t) d x\right|^{1 /(1-\sigma)} \leq C\left[\|u\|_{p}^{r /(1-\sigma)}+\left\|u_{t}\right\|_{2}^{s /(1-\sigma)}\right]
$$

for $1 / r+1 / s=1$. We take $s=2(1-\sigma)$ to get $r /(1-\sigma)=2 /(1-2 \sigma)=p$ by virtue of $(2.29)$. Therefore, (2.49) becomes

$$
\left|\int_{\Omega} u u_{t}(x, t) d x\right|^{1 /(1-\sigma)} \leq C\left[\|u\|_{p}^{p}+\left\|u_{t}\right\|_{2}^{2}\right], \quad \forall t \geq 0
$$

Similarly we have

$$
\left|\int_{\Omega} u q(x, t) d x\right|^{1 /(1-\sigma)} \leq C\left[\|u\|_{p}^{p}+\|q\|_{2}^{2}\right], \quad \forall t \geq 0 .
$$

Finally, by noting that

$$
\begin{aligned}
L^{1 /(1-\sigma)}(t) & =\left(H^{1-\sigma}(t)+\varepsilon \int_{\Omega} u\left(u_{t}+\frac{\beta \tau}{\kappa} q\right)(x, t) d x\right)^{1 /(1-\sigma)} \\
& \leq C\left(H(t)+\left|\int_{\Omega} u u_{t}(x, t) d x\right|^{1 /(1-\sigma)}+\left|\int_{\Omega} u q(x, t) d x\right|^{1 /(1-\sigma)}\right) \\
& \leq C\left[H(t)+\|u\|_{p}^{p}+\left\|u_{t}\right\|_{2}^{2}+\|q\|_{2}^{2}\right], \quad \forall t \geq 0,
\end{aligned}
$$


210 Blowup in thermoelasticity with second sound

and combining it with (2.45), we obtain

$$
L^{\prime}(t) \geq a_{0} L^{1 /(1-\sigma)}(t), \quad \forall t \geq 0,
$$

where $a_{0}$ is a positive constant depending on $\varepsilon A_{0}$ and $C$. A simple integration of (2.53) over $(0, t)$ then yields

$$
L^{(p-2) /(p+2)}(t) \geq \frac{1}{L^{-(p-2) / 2}(0)-a_{0} t(p-2) / 2} .
$$

Therefore, $L(t)$ blows up in a time

$$
T^{*} \leq \frac{1-\alpha}{\alpha a_{0}[L(0)]^{(p-2) /(p+2)}} .
$$

Remark 2.4. The estimate (2.55) shows that the larger $L(0)$ is the quicker the blowup takes place.

\section{Acknowledgment}

The first author would like to express his sincere thanks to KFUPM for its support.

\section{References}

[1] M. Aassila, Nonlinear boundary stabilization of an inhomogeneous and anisotropic thermoelasticity system, Appl. Math. Lett. 13 (2000), no. 1, 71-76.

[2] C. M. Dafermos and L. Hsiao, Development of singularities in solutions of the equations of nonlinear thermoelasticity, Quart. Appl. Math. 44 (1986), no. 3, 463-474.

[3] W. J. Hrusa and S. A. Messaoudi, On formation of singularities in one-dimensional nonlinear thermoelasticity, Arch. Ration. Mech. Anal. 111 (1990), no. 2, 135-151.

[4] W. J. Hrusa and M. A. Tarabek, On smooth solutions of the Cauchy problem in one-dimensional nonlinear thermoelasticity, Quart. Appl. Math. 47 (1989), no. 4, 631-644.

[5] S. Jiang and R. Racke, Evolution Equations in Thermoelasticity, Chapman \& Hall/CRC Monographs and Surveys in Pure and Applied Mathematics, vol. 112, Chapman \& Hall/CRC, Florida, 2000.

[6] M. Kirane and N. Tatar, A nonexistence result to a Cauchy problem in nonlinear one dimensional thermoelasticity, J. Math. Anal. Appl. 254 (2001), no. 1, 71-86.

[7] S. A. Messaoudi, On weak solutions of semilinear thermoelastic equations, Rev. Maghrébine Math. 1 (1992), no. 1, 31-40.

[8] - A blowup result in a multidimensional semilinear thermoelastic system, Electron. J. Differential Equations 2001 (2001), no. 30, 1-9.

[9] Local existence and blow up in nonlinear thermoelasticity with second sound, Comm. Partial Differential Equations 27 (2002), no. 7-8, 1681-1693.

[10] S. A. Messaoudi and B. Said-Houari, Exponential stability in one-dimensional nonlinear thermoelasticity with second sound, To appear in Math. Meth. Appl. Sci.

[11] J. E. Muñoz Rivera and R. K. Barreto, Existence and exponential decay in nonlinear thermoelasticity, Nonlinear Anal. 31 (1998), no. 1-2, 149-162.

[12] J. E. Muñoz Rivera and M. L. Olivera, Stability in inhomogeneous and anisotropic thermoelasticity, Boll. Un. Mat. Ital. A (7) 11 (1997), no. 1, 115-127. 
[13] J. E. Muñoz Rivera and R. Racke, Smoothing properties, decay, and global existence of solutions to nonlinear coupled systems of thermoelastic type, SIAM J. Math. Anal. 26 (1995), no. 6, $1547-1563$.

[14] R. Racke, Blow-up in nonlinear three-dimensional thermoelasticity, Math. Methods Appl. Sci. 12 (1990), no. 3, 267-273.

[15] - Thermoelasticity with second sound-exponential stability in linear and non-linear 1-d, Math. Methods Appl. Sci. 25 (2002), no. 5, 409-441.

[16] Asymptotic behavior of solutions in linear 2- or 3-D thermoelasticity with second sound, Quart. Appl. Math. 61 (2003), no. 2, 315-328.

[17] R. Racke and Y. Shibata, Global smooth solutions and asymptotic stability in one-dimensional nonlinear thermoelasticity, Arch. Ration. Mech. Anal. 116 (1991), no. 1, 1-34.

[18] R. Racke and Y. Wang, Propagation of singularities in one-dimensional thermoelasticity, J. Math. Anal. Appl. 223 (1998), no. 1, 216-247.

[19] Z. Saouli, Etude d'un système hyperbolique non lineaire d'équations thermoélastiques unidimensionelles, Master's thesis, University of Batna, 1995.

[20] M. Slemrod, Global existence, uniqueness, and asymptotic stability of classical smooth solutions in one-dimensional nonlinear thermoelasticity, Arch. Ration. Mech. Anal. 76 (1981), no. 2, 97-133.

[21] M. A. Tarabek, On the existence of smooth solutions in one-dimensional nonlinear thermoelasticity with second sound, Quart. Appl. Math. 50 (1992), no. 4, 727-742.

[22] E. Vitillaro, Global nonexistence theorems for a class of evolution equations with dissipation, Arch. Ration. Mech. Anal. 149 (1999), no. 2, 155-182.

Salim A. Messaoudi: Mathematical Sciences Department, King Fahd University of Petroleum and Minerals, Dhahran 31261, Saudi Arabia

E-mail address: messaoud@kfupm.edu.sa

Belkacem Said-Houari: Département de Mathématiques, Université Badji Mokhtar, BP 12, Annaba 23000, Algeria

E-mail address: saidhouarib@yahoo.fr 


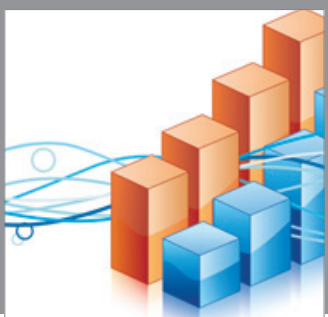

Advances in

Operations Research

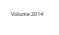

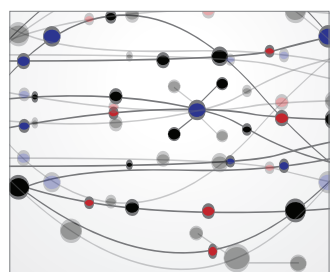

\section{The Scientific} World Journal
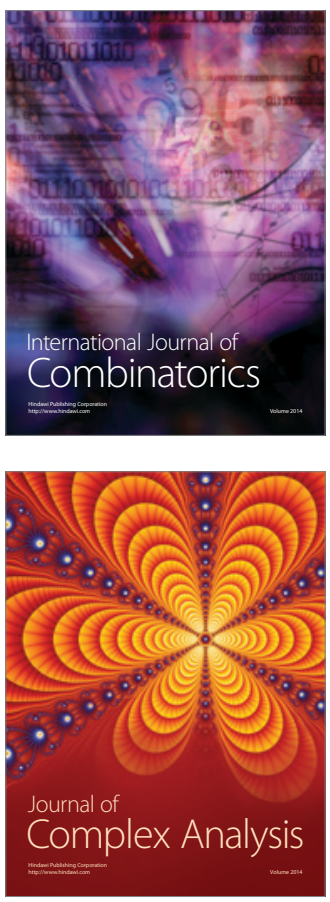

International Journal of

Mathematics and

Mathematical

Sciences
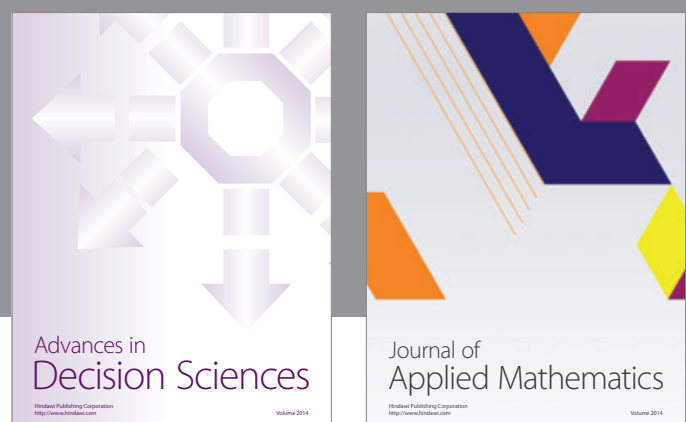

Journal of

Applied Mathematics
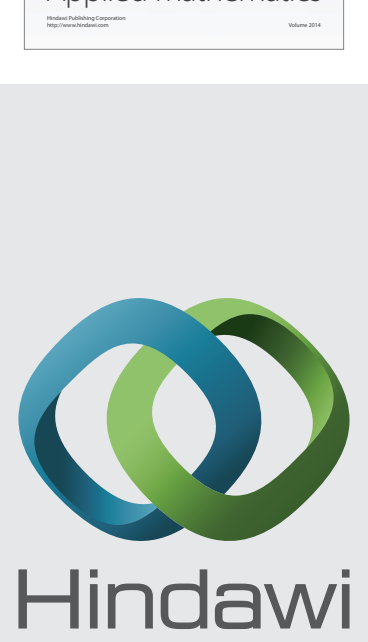

Submit your manuscripts at http://www.hindawi.com
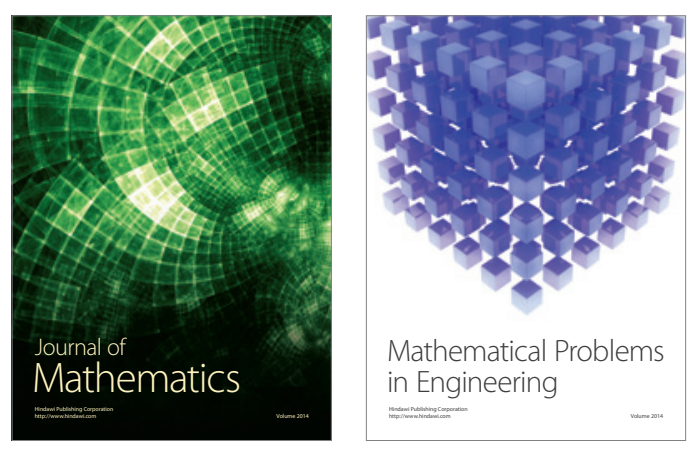

Mathematical Problems in Engineering
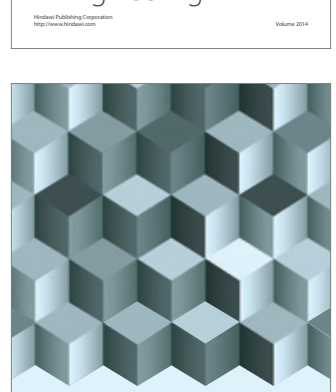

Journal of

Function Spaces
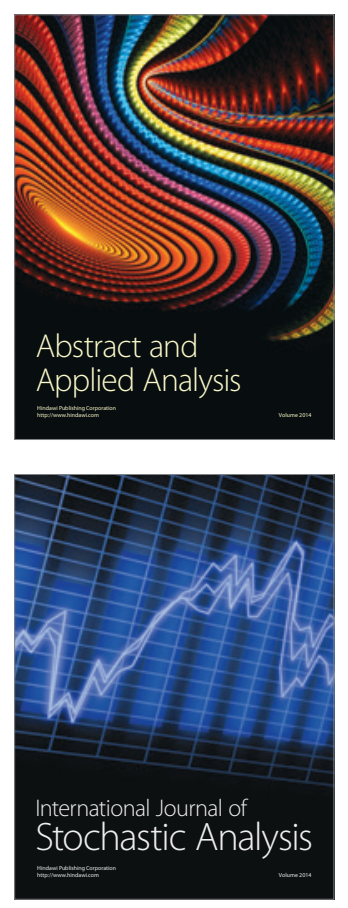

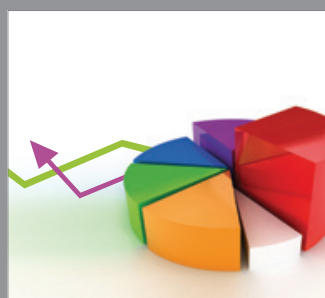

ournal of

Probability and Statistics

Promensencen
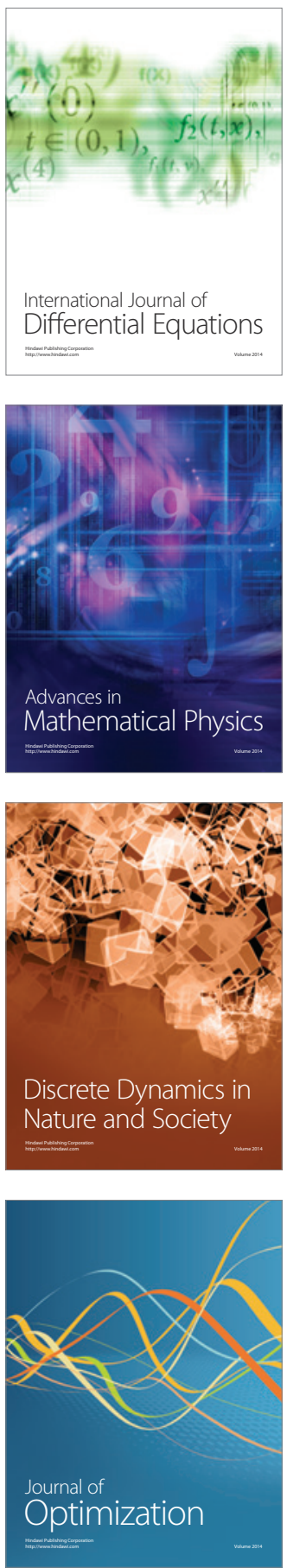